\title{
Self-calibration and Motion Recovery from Silhouettes with Two Mirrors
}

\author{
Hui Zhang ${ }^{1,2}$, Ling Shao ${ }^{3}$ and Kwan-Yee K. Wong ${ }^{4}$ \\ ${ }^{1}$ Dept. of Computer Science, United International College, \\ 28, Jinfeng Road, Tangjiawan, Zhuhai, Guangdong, China. \\ 2 Shenzhen Key Lab of Intelligent Media and Speech, \\ PKU-HKUST Shenzhen Hong Kong Institution, Shenzhen, China \\ ${ }^{3}$ Dept. of Electronic and Electrical Engineering, \\ The University of Sheffield, United Kingdom. \\ ${ }^{4}$ Dept. of Computer Science, The University of Hong Kong, \\ Pokfulam Road, Hong Kong
}

\begin{abstract}
This paper addresses the problem of self-calibration and motion recovery from a single snapshot obtained under a setting of two mirrors. The mirrors are able to show five views of an object in one image. In this paper, the epipoles of the real and virtual cameras are firstly estimated from the intersection of the bitangent lines between corresponding images, from which we can easily derive the horizon of the camera plane. The imaged circular points and the angle between the mirrors can then be obtained from equal angles between the bitangent lines, by planar rectification. The silhouettes produced by reflections can be treated as a special circular motion sequence. With this observation, technique developed for calibrating a circular motion sequence can be exploited to simplify the calibration of a single-view two-mirror system. Different from the state-of-the-art approaches, only one snapshot is required in this work for self-calibrating a natural camera and recovering the poses of the two mirrors. This is more flexible than previous approaches which require at least two images. When more than a single image is available, each image can be calibrated independently and the problem of varying focal length does not complicate the calibration problem. After the calibration, the visual hull of the objects can be obtained from the silhouettes. Experimental results show the feasibility and the preciseness of the proposed approach.
\end{abstract}

\section{Introduction}

Mirrors have been used for generating multiple views of an object, from which the visual hull can be obtained to recover the object shape and it has may applications [18] [14] [19]. The object and its reflections generally provide symmetric relationships for recovering parameters of the camera and the mirror [24] [5] (or a pair of mirrors [4]). In [7], Gluckman and Nayar discussed the geometry and calibration of a two-mirror system using point correspondences. $\mathrm{Hu}$ et. al. [10] later presented an approach for obtaining the camera calibration from the 
constraints imposed by both the silhouette outlines and point correspondences. Fujiyama et. al. [5] clearly presented the geometry of multiple view using one mirror. Forbes et. al. [3] introduced an approach based on silhouettes alone. However, they assumed an orthographic projection model and required a dense search in the parameter space to determine the initial estimates. Later in [2] they improved their method by providing closed form solutions for the initial parameter estimates using a perspective camera model. However, at least two snapshots were required for acquiring the calibration and estimating the motion. Besides, their method still required the assumption of an orthographic projection in the process of motion recovery. In another recent work, Huang [11] proved that the focal length can be recovered from a single snapshot of the setting, but it was based on the assumption that the principal point lied on the image center.

By exploring the geometry of two mirrors, this paper relates a two-mirror setting to a circular motion. Many studies have been conducted in circular motion [17], [1], [12], [16], [9]. Traditional method obtained the rotation angles by careful calibration [17], i.e., the camera internal parameters, rotation angles, camera orientations, etc are all accurately known. In [1], Fitzgibbon et al. developed a method to handle the case of uncalibrated camera with unknown rotation angles based on a projective reconstruction. Their method is based on the projective geometry of single axis motion, and it involves the computation of both fundamental matrices and trifocal tensors from point correspondences. Jiang et al. [12] further extended this approach by making use of the conic trajectories of the rotating point features, and developed an algorithm that requires neither the computation of fundamental matrices nor trifocal tensors. An alternative approach is to exploit the silhouettes of the object. Mendonça et al. [16] proposed to recover the structure and motion in several steps, each of which only involves a low dimensional optimization. However, the camera intrinsics are still required in the procedure for recovering the rotation angles and the subsequent Euclidean reconstruction. Zhang et al. [20] introduced an approach for uncalibrated silhouettes based on a new formulation of the circular point, and they further extended their method by making use of the 1D camera geometry [21].

Inspired by [16] and [20], it is derived in this paper that circular motions of a pair of symmetric objects can be obtained from the relationships between the image of the object and its reflections in two mirrors. The silhouettes produced by reflections can be treated as a special circular motion sequence. With this observation, technique developed for calibrating a circular motion sequence can be exploited to simplify the calibration of a single-view two-mirror system. Different from the state-of-the-art techniques [2] which assume orthogonal projection for recovering the motion, this work is totally based on perspective projection and hence it is applicable for any real scenes. More importantly, only one snapshot is required in this work for calibrating a natural camera (with three unknowns) and recovering the motion. This is more flexible than the previous approaches which require at least two images and the problem of varying focal length in multiple views will not complicate the calibration problem. Experimental results show the feasibility and the preciseness of the proposed approach. 
The remainder of the paper is organized as follows. Section 2 gives the fundamental theories of the two-mirror setup. It also presents the relationship between the two-mirror setting and the circular motion. Section 3 describes selfcalibration of the camera, with the recovery of image invariants, i.e., the circular points, the imaged rotation axis, the vanishing point of the $\mathrm{x}$-axis of the real camera and the mirror angles, etc. Section 4 introduces implementation details of the proposed technique. Section 5 presents the experimental results, followed by discussions and conclusions in Section 6 .

\section{Two-Mirror Setup and Circular Motion}

\section{$2.1 \quad$ Two-Mirror Setup}

In this section, we introduce the two-mirror setup in a 3D space. The reflections shown by mirrors are used to derive vanishing points for parallel tangent lines and these vanishing points all lie on the vanishing line $\mathbf{l}_{h}$ of a plane in which the real and virtual cameras lie.

Let us first consider a camera $C$ capturing an object $O$ and its reflection $O_{1}$ in a mirror $M$ (see Fig.1(a)). Note that there would be a virtual camera $C_{1}$ which is the reflection of $C$ in the mirror $M$. Consider two planes $\Pi_{\top}$ and $\Pi_{\perp}$ passing through the two cameras $C, C_{1}$ and tangent to both $O$ and $O_{1}$ externally. As both sides of the mirror are symmetric, the tangent points on $O$ and $O_{1}$, i.e., $\mathbf{X}, \mathbf{X}_{1}$ and $\mathbf{Y}, \mathbf{Y}_{1}$, provide two point correspondences with respect to the mirror. The joint lines $\mathbf{X X}_{\mathbf{1}}, \mathbf{Y Y}_{1}$ and the line joining the camera centers $C C_{1}$ are parallel to each other and perpendicular to the mirror plane. Let the images of $\mathbf{X X}_{\mathbf{1}}$ and $\mathbf{Y} \mathbf{Y}_{1}$ in the real camera $C$ be $\mathbf{l}_{\top}, \mathbf{l}_{\perp}$, respectively, which are the bitangents to the silhouettes of $O$ and $O_{1}$. Their intersection point $\mathbf{v}_{1}$ indicates the vanishing point of the perpendicular direction of the mirror plane.

Now let us consider the two-mirror setup (see Fig.1(b)) capturing five objects. The camera $C$ observes the real object $O$ and also its four mirror reflections $O_{1}$, $O_{2}, O_{12}$ and $O_{21}$. The virtual object $O_{1}$ is the reflection of $O$ in the mirror $M_{1}$; $O_{2}$ is the reflection of $O$ in the mirror $M_{2} ; O_{12}$ is the reflection of $O_{1}$ in the mirror $M_{2}$; and $O_{21}$ is the reflection of $O_{2}$ in the mirror $M_{1}$. Note there are two virtual mirrors $M_{v 1}, M_{v 2}$ which reflect $O_{1}$ to $O_{21}, O_{2}$ to $O_{12}$, respectively. There are also several virtual cameras which are the reflections of the real camera $C$, i.e., the virtual cameras $C_{1}, C_{2}$ (the reflection of $C$ in the mirror $M_{1}, M_{2}$, respectively), the virtual camera $C_{21}$ (the reflection of $C_{1}$ in the mirror $M_{v 1}$ and also the reflection of $C_{2}$ in $M_{1}$ ), the virtual camera $C_{12}$ (the reflection of $C_{2}$ in the mirror $M_{v 2}$ and also the reflection of $C_{1}$ in $M_{2}$ ). Note all the cameras lie on a common plane $\Pi$ and the bitangents $\mathbf{X X}_{\mathbf{1}}, \mathbf{Y} \mathbf{Y}_{1}$ in Fig.1(a) are parallel to $\Pi$, which implies the five (real and virtual) objects lie on a plane parallel to $\Pi$. Besides, note that the mirrors $M_{1}, M_{2}, M_{v 1}, M_{v 2}$ intersect along a common line $\mathbf{L}_{s}$ which is perpendicular to $\Pi$.

Let the images of $O, O_{1}, O_{2}, O_{21}, O_{12}$ be $o, o_{1}, o_{2}, o_{21}, o_{12}$, respectively, and the vanishing line of $\Pi$ be $\mathbf{l}_{h}$ (see Fig.1(c)). From the mirror reflections, it can 


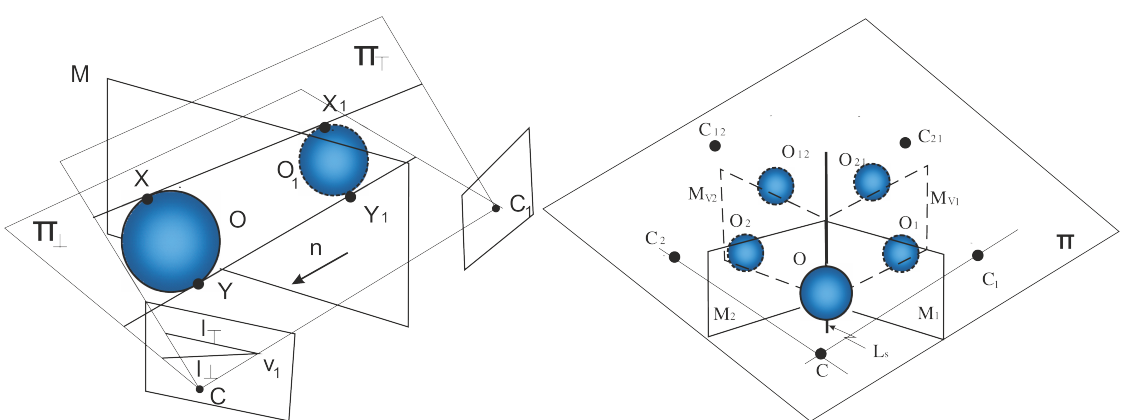

(a)

(b)

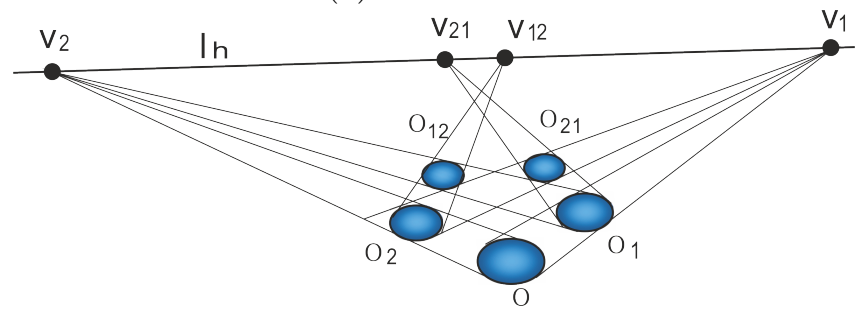

(c)

Fig. 1. Geometry of the mirror(s). (a) One mirror setup. (b) Two mirror setup. (c) The image of the two-mirror setup.

be seen that the outer bitangents of $o, o_{1}$ and that of $o_{2}, o_{21}$ intersect with the horizon $\mathbf{l}_{h}$ at the vanishing point $\mathbf{v}_{1}$; the outer bitangents of $o, o_{2}$ and that of $o_{1}, o_{12}$ intersect with $\mathbf{l}_{h}$ at $\mathbf{v}_{2}$; the outer bitangents of $o_{1}, o_{21}$ intersect with $\mathbf{l}_{h}$ at $\mathbf{v}_{21}$; the outer bitangents of $o_{2}, o_{12}$ intersect with $\mathbf{l}_{h}$ at $\mathbf{v}_{12}$. Hence the horizon $\mathbf{l}_{h}$ can be recovered as a line passing through all the vanishing points $\mathbf{v}_{1}, \mathbf{v}_{2}, \mathbf{v}_{21}$ and $\mathbf{v}_{12}$.

\subsection{Relating the Two Mirror Setting to the Circular Motion}

We have observed that the silhouettes produced by reflections can be treated as a special circular motion sequences. In this section, we will illustrate this in detail. Consider the top view of Fig.1(b). The real and virtual cameras $C, C_{1}$, $C_{2}, C_{21}, C_{12}$ are all on the plane $\Pi$. Let the real camera $C$ lie on the negative $Z$ axis of the world coordinate system and the mirror intersection line $\mathbf{L}_{s}$ coincides with the $Y$-axis (see Fig.2(a)).The projection matrix of $C$ is

$$
\mathbf{P}_{C}=\mathbf{K R}[\mathbf{I} \mid-\mathbf{T}]
$$

where $\mathbf{K}$ is the camera intrinsic matrix, $\mathbf{R}$ is the camera initial orientation and $\mathbf{T}=\left[\begin{array}{lll}0 & 0 & -1\end{array}\right]^{T}$ is the camera center. Let the angle between the mirror $M_{1}$ and the negative $Z$-axis be $\sigma$, and the angle between the mirror $M_{2}$ and the negative $Z$-axis be $\varphi$. Then the angle between $M_{1}$ and $M_{2}$ is $\theta=\sigma+\varphi$. From the mirror 
reflections, it can be seen that $|O C|=\left|O C_{1}\right|=\left|O C_{2}\right|=\left|O C_{21}\right|=\left|O C_{21}\right|$, where $|A B|$ indicates the length of $A B$. Hence the camera centers $C, C_{1}, C_{2}, C_{21}, C_{12}$ lie on a circle (see Fig.2(a)). Besides, note that the angle between $M_{1}$ and $O C_{1}$ is $\sigma$, and the angle between $M_{2}$ and $O C_{2}$ is $\varphi$. Similarly, the other angles between virtual cameras and mirrors can also be easily derived as shown in Fig.2(a).

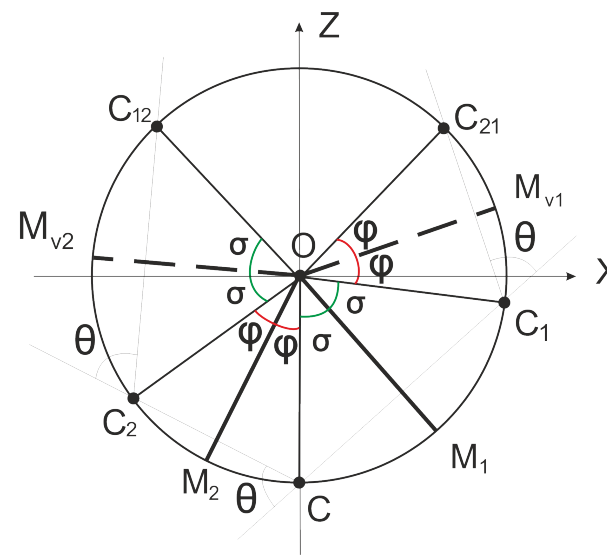

(a)

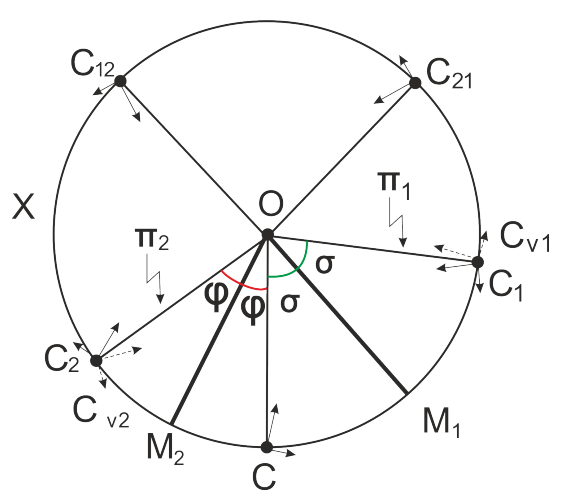

(b)

Fig. 2. Top view of the mirror setup. (a) The camera centers lie on a circle. (b) The cameras perform circular motion.

Imagine that there is a plane mirror $\Pi_{1}$ which passes through $\mathbf{L}_{s}$ and $C_{1}$. Let $C_{v 1}$ be the reflection of $C_{1}$ according to $\Pi_{1}$. The camera projection matrices for $C_{1}$ and $C_{v 1}$ can be represented by

$$
\begin{aligned}
& \mathbf{P}_{\mathbf{C}_{1}}=\mathbf{K R}\left[\mathbf{R}_{\mathbf{Y}}(\sigma) \sum \mathbf{R}_{\mathbf{Y}}(-\sigma) \mid-\mathbf{T}\right], \\
& \mathbf{P}_{\mathbf{C}_{\mathbf{v} 1}}=\mathbf{K R}\left[\mathbf{R}_{\mathbf{Y}}(\mathbf{2} \sigma) \mid-\mathbf{T}\right],
\end{aligned}
$$

where $\sum=\operatorname{diag}([-111]), \mathbf{R}_{Y}(\sigma)$ indicate rotation around $Y$-axis by an angle $\sigma$. Similarly, let $C_{v 2}$ be the reflection of $C_{2}$ according to $\Pi_{2}$, where $\Pi_{2}$ is a virtual plane mirror passing through $\mathbf{L}_{s}$ and $C_{2}$. We can easily derive the projection matrices for $C_{2}$ and $C_{v 2}$ in a similar way.

Now it can be easily observed that $C_{21}$ is obtained by rotating $C$ counterclockwise about the point $O$ with an angle $2(\varphi+\sigma)$, i.e., twice of the angle $\theta$ between the mirror $M_{1}$ and $M_{2}$. Similarly, $C_{12}$ is obtained by rotating $C$ clockwise about the point $O$ with $2 \theta . C_{2}$ is obtained by rotating $C_{1}$ clockwise about the point $O$ with $2 \theta$. Therefore, it can be observed that $C, C_{v 1}, C_{21}$, $C_{12}, C_{v 2}$ are the cameras performing a circular motion and the rotation axis is the $Y$-axis. Besides, it can also be derived that the angles have the following constraints

$$
\angle C C_{1} C_{21}=\angle C_{1} C C_{2}=\angle C C_{2} C_{12}=\pi-\theta .
$$


Under circular motion, the fundamental matrix relating any two views can be explicitly parameterized in terms of the image invariants, and is given by [1][15]

$$
\mathbf{F}(\psi)=\left[\mathbf{v}_{x}\right]_{\times}+\kappa \tan \frac{\psi}{2}\left(\mathbf{l}_{s} \mathbf{l}_{h}^{T}+\mathbf{l}_{h} \mathbf{l}_{s}^{T}\right),
$$

where $\psi$ is the rotation angle between the two views. $\mathbf{l}_{s}$ is the imaged rotation axis and $\mathbf{v}_{x}$ is the vanishing point of $X$-axis. $\kappa$ is an unknown but fixed scalar used to account for the different scales used in the homogeneous representations of the two terms in the summation [22].

\section{Self-Calibration of Two-Mirror Setting}

In this section, a novel approach for self-calibrating the two-mirror setup will be introduced. The imaged circular points of the horizontal camera plane are firstly derived by metric rectification of the horizontal plane. The angle between the mirrors can thus be easily obtained. From the metric rectification, the imaged rotation axis can be derived. The vanishing point of the $X$-axis can thus be obtained by a cross ratio relationship. These image invariants could be used for a camera self-calibration.

\subsection{Recovery of the Circular Point and the Mirror Angle}

First, from the horizon $\mathbf{l}_{h}=\left[\begin{array}{lll}l_{1} & l_{2} & l_{3}\end{array}\right]^{T}$ estimated in Section 2, the image in Fig.1(b) can be rectified to an affine plane using a 'pure projective' transformation [13], given by

$$
\mathbf{P}=\left[\begin{array}{ccc}
1 & 0 & 0 \\
0 & 1 & 0 \\
l_{1} & l_{2} & l_{3}
\end{array}\right] .
$$

Let the circular points be $[\alpha \mp j \beta, 1,0]^{T}$ on the affine plane, the plane can be further transformed to a metric plane using an affine transformation [13] given by

$$
\mathbf{A}=\left[\begin{array}{ccc}
\frac{1}{\beta} & -\frac{\alpha}{\beta} & 0 \\
0 & 1 & 0 \\
0 & 0 & 1
\end{array}\right]
$$

Note that in equation (3) the angle $\angle C C_{1} C_{21}$ formed by the line $\mathbf{l}_{C C_{1}}=$ $\left[\begin{array}{lll}l_{a 1} & l_{a 2} & l_{a 3}\end{array}\right]^{T}$ and $\mathbf{l}_{C_{1} C_{21}}=\left[\begin{array}{lll}l_{b 1} & l_{b 2} & l_{b 3}\end{array}\right]^{T}$, and the angle $\angle C_{1} C C_{2}$ formed by $\mathbf{l}_{C_{1} C}=$ $\left[\begin{array}{lll}l_{p 1} & l_{p 2} & l_{p 3}\end{array}\right]^{T}$ and $\mathbf{l}_{C C_{2}}=\left[\begin{array}{lll}l_{q 1} & l_{q 2} & l_{q 3}\end{array}\right]^{T}$ are equal unknown angles on the world plane. Hence the $2 \mathrm{D}$ point $(\alpha, \beta)$ can be shown lying on the circle with center on the point $\left(c_{\alpha}, c_{\beta}\right)=\left(\frac{a q-b p}{a-b-p+q}, 0\right)$ and squared radius $r^{2}=\left(\frac{a q-b p}{a-b-p+q}\right)^{2}+$ $\frac{(a-b)(a b-p q)}{a-b-p+q}-a b$, where $a=-\frac{l_{a 2}}{l_{a 1}}, b=-\frac{l_{b 2}}{l_{b 1}}, p=-\frac{l_{p 2}}{l_{p 1}}$ and $q=-\frac{l_{q 2}}{l_{q 1}}$ indicate the directions of each line. Similarly, by making use an additional unknown equal angle $\angle C C_{2} C_{12}$ in equation $(3),(\alpha, \beta)$ can be determined easily. Hence the pair of circular points in the original image can be recovered, by $\mathbf{i}, \mathbf{j}=[(\alpha \pm$

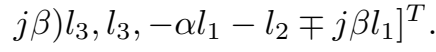


From $\mathbf{i}, \mathbf{j}$, the angle between the two mirrors can thus be directly obtained by using the Laguerre's formula

$$
\theta=\frac{1}{2 j} \log \left\{\mathbf{v}_{\mathbf{1}}, \mathbf{v}_{\mathbf{2}} ; \mathbf{i}, \mathbf{j}\right\}
$$

where $\left\{\mathbf{v}_{\mathbf{1}}, \mathbf{v}_{\mathbf{2}} ; \mathbf{i}, \mathbf{j}\right\}$ denotes a cross-ratio, $j^{2}=-1$.

\subsection{Recovery of the Imaged Rotation Axis}

By making use of the projection and affine transformations $\mathbf{P}$ and $\mathbf{A}$ (see Section 3.1), the imaged circular points $\mathbf{i}, \mathbf{j}$ are expected to be rectified to their genuine position $\mathbf{I}, \mathbf{J}=\left[\begin{array}{lll}1 \pm j & 0\end{array}\right]^{T}$. However, we still need a rotation $\mathbf{R}$ to transform the imaged circular points to their genuine position. Hence by the same transformations, the imaged rotation axis $\mathbf{l}_{s}$ can be rectified to a plane $\left[\begin{array}{lll}1 & 0 & 0\end{array}\right]^{T}$ passing through the camera center and the rotation axis. Thus $\mathbf{l}_{s}$ can be initialized as

$$
\mathbf{l}_{s}=(\mathbf{R A P})^{T}\left[\begin{array}{l}
1 \\
0 \\
0
\end{array}\right] .
$$

The vanishing point $\mathbf{v}_{z}$ of the $Z$-axis can be obtained as the intersection between $\mathbf{l}_{s}$ and $\mathbf{l}_{h}$. The vanishing point $\mathbf{v}_{x}$ can also be easily recovered from the cross ratio

$$
\left\{\mathbf{i}, \mathbf{j} ; \mathbf{v}_{x}, \mathbf{v}_{z}\right\}=-1 .
$$

The angle $\sigma$ between the mirror $M_{1}$ and the negative $Z$-axis (see Fig.2(a)) can be obtained by $\sigma=\pi / 2-\log \left\{\mathbf{v}_{1}, \mathbf{v}_{z} ; \mathbf{i}, \mathbf{j}\right\} /(2 j)$ and the angle $\varphi$ between the mirror $M_{2}$ and the negative $Z$-axis can be obtained by $\varphi=\pi / 2-\log \left\{\mathbf{v}_{2}, \mathbf{v}_{z} ; \mathbf{i}, \mathbf{j}\right\} /(2 j)$, where $j^{2}=-1$.

Note the pair of circular points $\mathbf{i}, \mathbf{j}$ of the circular plane are given by [22]

$$
\mathbf{i}, \mathbf{j} \sim \mathbf{v}_{x} \mp j \kappa \mathbf{l}_{s} \times \mathbf{l}_{h},
$$

where $\kappa$ is the same scalar in equation (4). As $\mathbf{i}, \mathbf{j}, \mathbf{v}_{x}, \mathbf{l}_{s}, \mathbf{l}_{h}$ are known variables, $\kappa$ can be easily obtained. Hence the epipoles $\mathbf{e}_{i}(i=1,2)$ between a pair of the images of the circular motion can be obtained from [15]

$$
\mathbf{e}_{i} \sim \mathbf{v}_{x}-(-1)^{i} \kappa \tan \frac{\psi}{2} \mathbf{l}_{s} \times \mathbf{l}_{h}
$$

And the refinement of the imaged rotation axis $\mathbf{l}_{s}$ can be carried out as a two dimensional optimization problem by minimizing the distance between the transformed epipolar tangents $\mathbf{l}_{i}^{\prime}$ and the silhouette in the second image (see Fig.3). The transformation is defined by a harmonic homology [8][15] $\mathbf{W}^{-T}$, which is given by $\mathbf{W}=\mathbf{I}-2 \frac{\mathbf{v}_{x} \mathbf{l}_{s}^{T}}{\mathbf{v}_{x}^{T} \mathbf{s}_{s}}$. 


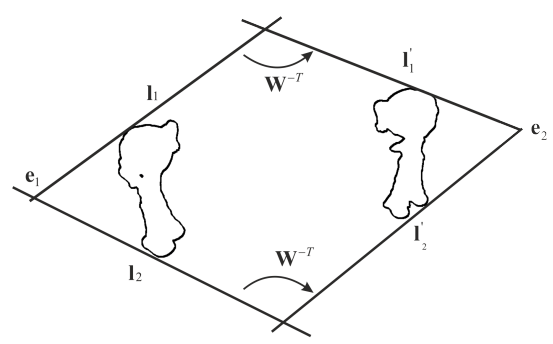

Fig. 3. The overlapping of two silhouettes and their epipolar tangents under the circular motion. $\mathbf{l}_{1}, \mathbf{l}_{1}^{\prime}, \mathbf{l}_{2}$ and $\mathbf{l}_{2}^{\prime}$ are the outer epipolar tangent lines.

\subsection{Self-Calibration and Motion Recovery}

The obtained imaged circular points can be used to find the camera intrinsics since they lie on the image of the absolute conic (IAC) $\omega$. Besides, the imaged rotation axis $\mathbf{l}_{s}$ and the vanishing point $\mathbf{v}_{x}$ define a pole-polar relationship w.r.t. $\omega[8] . \omega$ can then be estimated from the following constraints:

$$
\left\{\begin{array}{l}
\mathbf{i}^{T} \omega \mathbf{i}=0 \text { and } \mathbf{j}^{T} \omega \mathbf{j}=0 \\
\mathbf{l}_{s}=\omega \mathbf{v}_{x}
\end{array}\right.
$$

Since these provide three independent constraints, given only a single image, a natural camera with zero skew and unit aspect ratio can be calibrated by Cholesky decomposition [6] of $\omega$. For multiple images captured with varying focal length, each image can be calibrated independently. Hence the problem of varying focal length does not complicate the calibration problem.

\section{Implementation}

Here we introduce using one snapshot to calibrate the camera and recover the motion. Cubic B-spline snakes are used to extract silhouettes from the images with sub-pixel localization accuracy. The horizon $\mathbf{l}_{h}$ is initially obtained by robustly fitting a line to the vanishing points constructed from the outer tangents to the object silhouettes in the image. $\mathbf{l}_{h}$ and the vanishing points are then refined by minimizing the distance between the tangent lines and the corresponding silhouettes.

The image can then be transformed to an affine plane by equation (5). Then the imaged circular points $\mathbf{i}, \mathbf{j}$ can be obtained by making use equal unknown angles in the world plane (see Section 3.1 in detail). The imaged rotation axis $\mathbf{l}_{s}$ is then initialized as the rectified $Y Z$-plane by equation (8) and the vanishing point $\mathbf{v}_{x}$ of $X$-axis can be recovered by (9). $\mathbf{l}_{s}$ and $\mathbf{v}_{x}$ can be refined by the finding of a line tangent to one silhouette which is transformed by the harmonic homology $\mathbf{W}^{-T}$ to a line tangent to another silhouette under the circular motion [16]. From the estimated $\mathbf{l}_{h}, \mathbf{i}, \mathbf{j}, \mathbf{l}_{s}, \mathbf{v}_{x}$, the fixed scalar $\kappa$ and the rotation angles 
can be easily derived (see Section 3.2 for detail). Besides, a natural camera can be calibrated with the recovered $\mathbf{l}_{s}, \mathbf{v}_{x}$ and $\mathbf{i}, \mathbf{j}$, by equation (12). The camera extrinsic parameters can then be estimated by aligning the images of the horizon and the rotation axis through rectifying each image independently by a homography induced by a rotation about the camera center such that $\mathbf{L}_{\mathbf{s}}$ coincides with the $Y$-axis of the world coordinate and the $Z$-axis of the camera world coordinate coincides with the $Z$-axis of the world coordinate [?].

Besides, if multiple snapshots were taken, we need to specify the five silhouettes from different views in a common reference frame to refine the estimation. This can be achieved by firstly aligning the world coordinate recovered with different snapshots and rectifying the five-view silhouette sets with the camera matrices so that the cameras all point towards the rotation axis $\mathbf{L}_{\mathbf{s}}$. The silhouette sets are then scaled and translated along the rotation axis so that the outer epipolar tangents coincide with the projected tangents from silhouettes in the other silhouette set.

Finally, a bundle-adjustment using Levenberg Marquardt minimization is applied to refine all the parameters. The intrinsics and the angle $\theta$ between mirrors $M_{1}$ and $M_{2}$ are then estimated with the optimized entities, followed by a constructing the visual hull from silhouettes.

\section{$5 \quad$ Experiments and Results}

Real experiments were carried out to test the feasibility of the approach. The first experiment consisted views of a girl (see the first column Fig.4). The image had a resolution of $1296 \times 861$. Provided with only one single snapshot, the camera was self-calibrated under the assumption of a natural camera (zero-skew and unit aspect ratio). Column 2-4 of Table 1(a) compare the estimated camera matrix and the recovered mirror angle with that of the ground-truth (obtained with a planar calibration pattern [23]) and the approach introduced in [2]. It can be seen that the recovered angle $\theta$ between the mirrors has a high resolution. The focal length $f$ and the $u_{0}$ coordinate of the principal point were both precisely estimated while $v_{0}$ was not. This is due to the error in the estimated $\mathbf{v}_{x}$. Column 2-4 of Table 1 (b) show the experimental results with two snapshots. It can be seen the calibration results is better with more snapshots involved in estimation. From the recovered motion, Fig.4(c) shows the 3D model reconstructed with only one single snapshot and Fig.4(d) shows that with two snapshots. The model becomes more accurate may due to the reason that more snapshots may provide more accuracy in the camera calibration and the visual hull.

The second experiment consisted views of a monster (see the second colum of Fig.4). The image had a resolution of $1296 \times 861$. With only one single snapshot, column 6-9 of Table 1(a) compare the estimated camera matrix and the mirror angle with that of the ground-truth (obtained with a planar calibration pattern [23]) and the approach introduced in [2]. Column 6-9 of Table 1(b) show the result with two snapshots. From the estimated motion, Fig.4(d) shows the 3D 
(a)

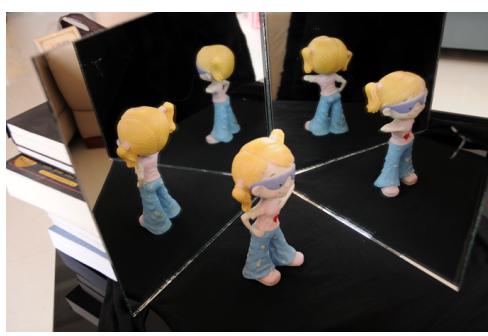

(b)

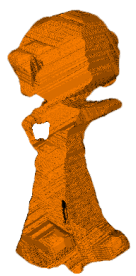

(c)

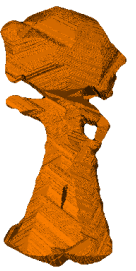

(d)
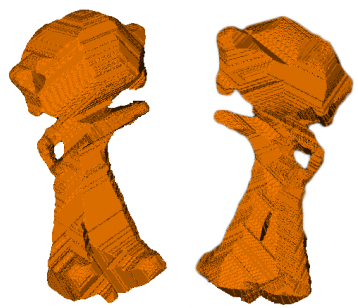

(f)
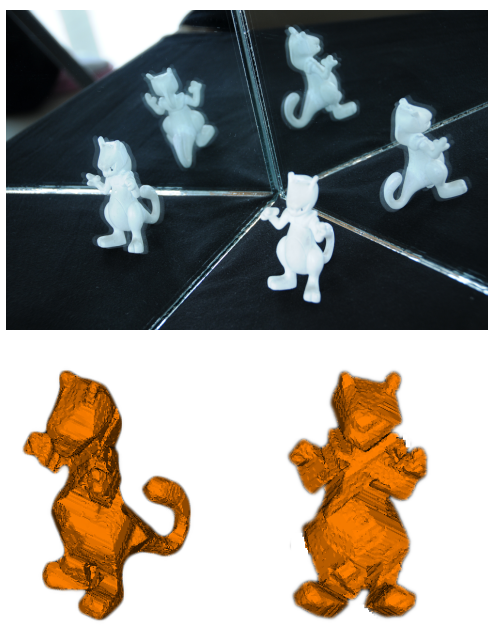

(e)
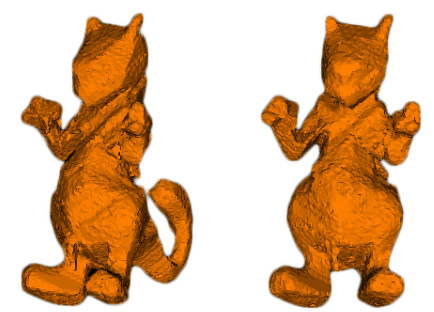

Fig. 4. Real experiment. The 1st column is for the experiments of the girl and the 2nd column is for that of the little monster. (a)\&(b) An image of the two mirror setting. (c)\&(d) The reconstructed 3D models from a snapshot. (e)\&(f) The reconstructed 3D models from 2 snapshots.

model reconstructed with only one single snapshot and Fig.4(f) shows that with two snapshots.

\section{Conclusions}

In this paper, we have presented a practical and efficient approach for selfcalibrating a camera from only a single snapshot obtained under a setting of two-mirror. We relate it with a circular motion and use image rectification to find the initial estimation of the imaged rotation axis. Different from the stateof-the-art approaches, only one snapshot is required in this work for calibrating a natural camera and recovering the motion. This is more flexible than the previous approaches which require at least two images. Hence the problem of varying focal length in multiple images does not complicate the calibration problem. After calibration, a visual hull of the object can be obtained from the silhouettes. 


\begin{tabular}{c|cccc|cccc}
\hline & \multicolumn{4}{|c|}{ Girl } & \multicolumn{4}{|c}{ Monster } \\
\hline- & $\mathrm{f}$ & $u_{0}$ & $v_{0}$ & $\theta$ & $\mathrm{f}$ & $u_{0}$ & $v_{0}$ & $\theta$ \\
\hline Ground-truth & 1178.4 & 663.78 & 440.74 & $74.3^{\circ}$ & 2971.4 & 623.89 & 415.31 & $74.3^{\circ}$ \\
Method in [2] & 1224.5 & 648 & 430.5 & - & 2950.6 & 648 & 430.5 & - \\
Proposed method & 1196.7 & 633.43 & 413.2 & $74.18^{\circ}$ & 2958.8 & 606.05 & 365.82 & $74.43^{\circ}$ \\
Percentage error to GT & $1.55 \%$ & $1.73 \%$ & $2.34 \%$ & $0.16 \%$ & $0.42 \%$ & $0.60 \%$ & $1.67 \%$ & $0.17 \%$
\end{tabular}

\begin{tabular}{c|cccc|cccc}
\hline \hline & \multicolumn{4}{|c|}{ Girl } & \multicolumn{4}{c}{ Monster } \\
\hline- & $\mathrm{f}$ & $u_{0}$ & $v_{0}$ & $\theta$ & $\mathrm{f}$ & $u_{0}$ & $v_{0}$ & $\theta$ \\
\hline Ground-truth & 1178.4 & 663.78 & 440.74 & $74.3^{\circ}$ & 2971.4 & 623.89 & 415.31 & $74.3^{\circ}$ \\
Method in [2] & 1173.2 & 646.9 & 432.71 & - & 2959.2 & 664.32 & 403.28 & - \\
Proposed method & 1172.7 & 648.97 & 426.45 & $74.35^{\circ}$ & 2976.9 & 659.46 & 375.4 & $74.21^{\circ}$ \\
Percentage error to GT & $0.48 \%$ & $1.26 \%$ & $1.21 \%$ & $0.07 \%$ & $0.18 \%$ & $1.20 \%$ & $1.34 \%$ & $0.12 \%$
\end{tabular}

Table 1. Comparative results of the intrinsic and the angle between the mirrors. Column 2-5 show experiments with images of a girl. Column 6-9 show experiments with images of a monster.(a) From a single snapshot. (b) From two views of the twomirror settings.

Experiments have produced convincing 3D models, demonstrating the practicality of our algorithm.

\section{Acknowledge}

The work is supported by the National Natural Science Foundation of China (Project no. 61005038) and an internal funding from United International College.

\section{References}

1. A. W. Fitzgibbon, G. Cross, and A. Zisserman. Automatic 3D model construction for turn-table sequences. In 3D Structure from Multiple Images of Large-Scale Environments, European Workshop SMILE'98, volume 1506, pages 155-170, Freiburg, Germany, June 1998.

2. K. Forbes, F. Nicolls, Jager G.D., and A. Voigt. Shape-from-silhouette with two mirrors and an uncalibrated camera. In Proc. 9th European Conf. on Computer Vision, pages 165-178, 2006.

3. K. Forbes, A. Voigt, and N. Bodika. Visual hulls from single uncalibrated snapshots using two plannar mirrors. In Proc. 15th South African Workshop on Pattern Recognition, 2004.

4. A.R.J. Francois, G.G. Medioni, and R. Waupotitsch. Reconstructing mirror symmetric scenes from a single view using 2-view stereo geometry. In International Conference on Pattern Recognition, volume 4, pages 12-16, 2002.

5. Shinji Fujiyama, Fumihiko Sakaue, and Jun Sato. Multiple view geometries for mirrors and cameras. International Conference on Pattern Recognition, pages 4548, Aug 2010.

6. J.E. Gentle. Numerical Linear Algebra for Applications in Statistics. SpringerVerlag, 1998. 
7. J.M. Gluckman and S.K. Nayar. Planar catadioptric stereo: Geometry and calibration. In Proc. Conf. Computer Vision and Pattern Recognition.

8. R.I. Hartley and A. Zisserman. Multiple View Geometry in Computer Vision. Cambridge University Press, Cambridge, UK, 2000.

9. C. Hernndez, F. Schmitt, and R. Cipolla. Silhouette coherence for camera calibration under circular motion. IEEE Trans. on Pattern Analysis and Machine Intelligence, 29(2):343-349, February 2007.

10. B. Hu, C.M. Brown, and Nelson R.C. Multiple-view 3-d reconstruction using a mirror. Technical Report TR863, May 2005.

11. P.H. Huang and S.H. Lai. Contour-based structure from reflection. In Proc. Conf. Computer Vision and Pattern Recognition, volume 1, pages 379-386, June 2006.

12. G. Jiang, H.T. Tsui, L. Quan, and A. Zisserman. Geometry of single axis motions using conic fitting. IEEE Trans. on Pattern Analysis and Machine Intelligence, 25(10):1343-1348, October 2003.

13. D. Liebowitz and A. Zisserman. Metric rectification from perspective images of planes. Proc. Conf. Computer Vision and Pattern Recognition, pages 482-488, 1998.

14. Gian Luca Mariottini, Stefano Scheggi, Fabio Morbidi, and Domenico Prattichizzo. Catadioptric stereo with planar mirrors:multiple-view geometry and camera localization. Visual Servoing via Advanced Numerical Methods, pages 3-21, 2010.

15. P. R. S. Mendonça, K.-Y. K. Wong, and R. Cipolla. Camera pose estimation and reconstruction from image profiles under circular motion. In Proc. 6th European Conf. on Computer Vision, volume 1843, pages 864-877, Ireland, June 2000.

16. P. R. S. Mendonça, K.-Y. K. Wong, and R. Cipolla. Epipolar geometry from profiles under circular motion. IEEE Trans. on Pattern Analysis and Machine Intelligence, 23(6):604-616, June 2001.

17. W. Niem. Robust and fast modelling of $3 \mathrm{~d}$ natural objects from multiple views. In SPIE Proceedings - Image and Video Processing II, volume 2182, pages 388-397, 1994.

18. Jurgen Rurainsky and Peter Eisert. Mirror-based multi-view analysis of facial motions. In Prof. of International Conference on Image Processing, volume 3, pages 73-76, 2007.

19. Brandon M. Smith, David G. Stork, and Li Zhang. Three-dimensional reconstruction from multiple reflected views within a realist painting: An application to scott frasers three way vanitas. The 21st Annual IST/SPIE Symposium on Electronic Imaging, 7239(7239), January 2009.

20. H. Zhang and K.-Y. K. Wong. Self-calibration of turntable sequences from silhouettes. IEEE Trans. on Pattern Analysis and Machine Intelligence, 31(1):5-14, January 2009.

21. H. Zhang, K.-Y. K. Wong, Zhang G., Liang C., and Zhang G. 1d camera geometry and its application to the self-calibration of circular motion sequences. IEEE Trans. on Pattern Analysis and Machine Intelligence, 30(12):2243-224, December 2008.

22. H. Zhang, G. Zhang, and K.-Y. K. Wong. Auto-calibration and motion recovery from silhouettes for turntable sequences. In Proc. British Machine Vision Conference, volume I, pages 79-88, Oxford, UK, September 2005.

23. Z.Y. Zhang. A flexible new technique for camera calibration. IEEE Trans. on Pattern Analysis and Machine Intelligence, 22(11):1330-1334, November 2000.

24. Z.Y. Zhang and H.T. Tsui. 3d reconstruction from a single view of an object and its image in a plane mirror. In International Conference on Pattern Recognition, volume II, pages 1174-1176, 1998 . 